\title{
Twist-2 relation and sum rule for tensor-polarized parton distribution functions of spin-1 hadrons
}

\section{S. Kumano ${ }^{a, b}$ and Qin-Tao Song ${ }^{c}$}

\author{
${ }^{a}$ KEK Theory Center, Institute of Particle and Nuclear Studies, \\ High Energy Accelerator Research Organization (KEK), \\ Oho 1-1, Tsukuba, Ibaraki, 305-0801, Japan \\ ${ }^{b} J-P A R C$ Branch, KEK Theory Center, Institute of Particle and Nuclear Studies, KEK, \\ and Theory Group, Particle and Nuclear Physics Division, J-PARC Center, \\ Shirakata 203-1, Tokai, Ibaraki, 319-1106, Japan \\ ${ }^{c}$ School of Physics and Microelectronics, Zhengzhou University, \\ Zhengzhou, Henan 450001, China
}

E-mail: shunzo.kumano@kek.jp, songqintao@zzu.edu.cn

ABstract: Sum rules for structure functions and their twist-2 relations have important roles in constraining their magnitudes and $x$ dependencies and in studying higher-twist effects. The Wandzura-Wilczek (WW) relation and the Burkhardt-Cottingham (BC) sum rule are such examples for the polarized structure functions $g_{1}$ and $g_{2}$. Recently, new twist3 and twist-4 parton distribution functions were proposed for spin- 1 hadrons, so that it became possible to investigate spin-1 structure functions including higher-twist ones. We show in this work that an analogous twist-2 relation and a sum rule exist for the tensorpolarized parton distribution functions $f_{1 L L}$ and $f_{L T}$, where $f_{1 L L}$ is a twist-2 function and $f_{L T}$ is a twist-3 one. Namely, the twist-2 part of $f_{L T}$ is expressed by an integral of $f_{1 L L}$ (or $b_{1}$ ) and the integral of the function $f_{2 L T}=(2 / 3) f_{L T}-f_{1 L L}$ over $x$ vanishes. If the parton-model sum rule for $f_{1 L L}\left(b_{1}\right)$ is applied by assuming vanishing tensor-polarized antiquark distributions, another sum rule also exists for $f_{L T}$ itself. These relations should be valuable for studying tensor-polarized distribution functions of spin- 1 hadrons and for separating twist-2 components from higher-twist terms, as the WW relation and BC sum rule have been used for investigating $x$ dependence and higher-twist effects in $g_{2}$. In deriving these relations, we indicate that four twist-3 multiparton distribution functions $F_{L T}, G_{L T}$, $H_{L L}^{\perp}$, and $H_{T T}$ exist for tensor-polarized spin-1 hadrons. These multiparton distribution functions are also interesting to probe multiparton correlations in spin-1 hadrons. In the near future, we expect that physics of spin-1 hadrons will become a popular topic, since there are experimental projects to investigate spin structure of the spin-1 deuteron at the 
Jefferson Laboratory, the Fermilab, the nuclotron-based ion collider facility, the electronion colliders in US and China in 2020's and 2030's.

Keywords: Deep Inelastic Scattering (Phenomenology)

ARXIV EPRINT: 2106.15849 


\section{Contents}

1 Introduction 1

2 Wandzura-Wilczek relation and Burkhardt-Cottingham sum rule 3

3 Parton distribution functions of spin-1 hadrons and their twist-2 relation and sum rule

3.1 Matrix elements of nonlocal operators and parton distribution functions 6

$\begin{array}{lll}3.2 & \text { Twist-3 matrix element and multiparton distribution functions } & 10\end{array}$

$\begin{array}{lll}3.3 & \text { Twist-2 relation and sum rule } & 15\end{array}$

$\begin{array}{lll}4 & \text { Summary } & 18\end{array}$

\section{Introduction}

High-energy spin physics has been one of exciting fields in hadron physics since the late 1980's for clarifying the origin of nucleon spin. In addition to longitudinally-polarized collinear structure functions, we investigate transverse-spin and three-dimensional (3D) structure functions nowadays. Furthermore, structure functions of spin-1 hadrons will be investigated extensively in the near future due to the existence of new tensor-polarized observables.

We have been investigating structure functions of spin-1 hadrons, especially on the spin-1 deuteron. There are four tensor-polarized structure functions $b_{1-4}$ for a spin-1 hadron in charged-lepton deep inelastic scattering [1,2]. The leading-twist functions $b_{1}$ and $b_{2}$ are related with each other by the Callan-Gross type relation $2 x b_{1}=b_{2}$, and other functions $b_{3}$ and $b_{4}$ are higher-twist ones. For finding the overall $x$-dependent functional form, there is a useful parton-model sum rule for $b_{1}[3,4]$. The $b_{1}$ measurement by the HERMES collaboration obtained a finite sum $\int d x b_{1} \neq 0$ [5], which indicated a finite tensor-polarized antiquark distribution. Possible tensor-polarized parton distribution functions (PDFs) were proposed for explaining the HERMES data [6]. In future, an accurate determination of $b_{1}$ will be made by the experiment at the Thomas Jefferson National Accelerator Facility (JLab) [7]. This $b_{1}$ project is interesting in the sense that the HERMES data are different from a conventional theoretical estimate based on a convolution description [8]. In addition, the gluon transversity $\Delta_{T} g$ exists for the spin-1 deuteron, although it does not exist for the spin-1/2 nucleons. This is also an interesting observable to find new hadron physics in the deuteron beyond the simple bound system of nucleons. It is expected to be measured by an JLab experiment [9-11]. These $b_{1}$ and $\Delta_{T} g$ measurements could be continued at the electron-ion collider (EIC) [12] and the Electron-ion collider in China (EicC) [13]. 
Such tensor-polarized structure functions can be also investigated at hadron accelerator facilities. In fact, the proton-deuteron Drell-Yan process could probe the tensorpolarized PDFs, especially the tensor-polarized antiquark distributions [14-16] and the gluon transversity $[17,18]$. It could be realized as a Fermilab-SpinQuest experiment [19] when a polarized-deuteron target becomes ready [20]. In addition, since the Nuclotronbased Ion Collider fAcility (NICA) will have a polarized-deuteron beam, the tensorpolarized structure functions and the gluon transversity will be investigated, for example, by observing $\mathrm{J} / \psi$ production $[21,22]$. These structure functions are interesting for investigating especially exotic aspects of hadron physics, such as hidden color [23] and non-nucleonic component [24] in the deuteron.

On the other hand, transverse-momentum-dependent parton distribution functions (TMDs) became one of hot topics in hadron physics. It is intended to understand not only basics 3D structure of hadrons but also to find explicit color degrees of freedom in terms of color flow. Because the color is confined in hadrons, it is not easy to find its explicit signature in observables. The TMDs are such quantities to probe the color directly [25]. For example, a color Aharonov-Bohm effect and color entanglement phenomena could be investigated by the TMDs. In addition, gluon condensates are now investigated with the understanding of gluon TMDs in the nucleons and nuclei.

Recently, we investigated the tensor-polarized TMDs for spin-1 hadrons and found 30 new TMDs at the twist 3 and twist 4 [25] in addition to the twist-2 functions [26-29]. Integrating the TMDs over the partonic transverse momentum, we also found that there are three new collinear PDFs at the twist 3 and twist 4. Therefore, including the twist-2 PDF, we have the collinear PDFs $f_{1 L L}, e_{L L}, f_{L T}$, and $f_{3 L L}$ for spin-1 hadrons. By considering this situation, the purposes of this work are the following.

(1) We derive a useful twist-2 relation and a sum rule for $f_{1 L L}$ and $f_{L T}$ in analogy to the Wandzura-Wilczek relation [30] and the Burkhardt-Cottingham sum rule [31] for the structure functions $g_{1}$ and $g_{2}$. Here, $f_{1 L L}$ is a twist- 2 function and $f_{L T}$ is a twist-3 one, and $f_{1 L L}$ is often used as the tensor-polarized structure function $b_{1}$.

(2) We show that four twist-3 multiparton distribution functions exist in a tensor-polarized spin-1 hadron.

(3) We show that the leading deviation of the twist-2 relation, namely the higher-twist term, is expressed by these twist-3 multiparton distribution functions, so that we try to obtain the full-decomposition expression of $f_{L T}$ into twist-2 and twist-3 terms.

For simply deriving the twist-2 relation and sum rule, the studies of the twist-3 multiparton distributions functions are not necessary. However, we investigate one more step to express the higher-twist term of $f_{L T}$ by the twist-3 multiparton distributions functions in this work.

In this paper, the Wandzura-Wilczek relation and Burkhardt-Cottingham sum rule are introduced by the operator-product-expansion formalism in section 2 . Then, the details are explained on our derivations of analogous relations for tensor-polarized PDFs in section 3. First, correlation functions and collinear PDFs are introduced, and the matrix element of a nonlocal vector operator is expressed by the tensor-polarized collinear PDFs in section 3.1. 
Next, the nonlocal operator is written in term of the gluon field tensor and it is related to the multiparton distribution functions in section 3.2. A useful twist-2 relation and a sum rule are derived for $f_{1 L L}$ and $f_{L T}$ in section 3.3. Our studies are summarized in section 4 .

\section{Wandzura-Wilczek relation and Burkhardt-Cottingham sum rule}

In investigating structure functions, there are useful sum rules and relations among them for finding their functional behavior. For example, there is a useful relation for the polarized structure function $g_{2}$, which exists in the spin- $1 / 2$ nucleons. Since we intend to derive a relation which is analogous to the Wandzura-Wilczek (WW) relation and also a sum rule like the Burkhardt-Cottingham (BC) sum rule, we introduce their outline within the formalism of operator product expansion.

The polarized distribution functions $g_{1 L}, g_{T}$, and $g_{3 L}$ are defined by the matrix element of a nonlocal operator as

$$
\begin{aligned}
& \int \frac{d\left(P^{+} \xi^{-}\right)}{2 \pi} e^{i x P^{+} \xi^{-}}\left\langle P, S\left|\bar{\psi}(0) \gamma^{\mu} \gamma_{5} \psi(\xi)\right| P, S\right\rangle_{\xi^{+}=0, \vec{\xi}_{T}=0} \\
& \quad=2 M_{N}\left[g_{1 L}(x) \bar{n}^{\mu} S \cdot n+g_{T}(x) S_{T}^{\mu}+g_{3 L}(x) \frac{M_{N}^{2}}{\left(P^{+}\right)^{2}} n^{\mu} S \cdot n\right] .
\end{aligned}
$$

Here, $M_{N}$ is the nucleon mass, $\psi$ is the quark field, the lightcone vectors $n$ and $\bar{n}$ are defined by

$$
n^{\mu}=\frac{1}{\sqrt{2}}(1,0,0,-1), \quad \bar{n}^{\mu}=\frac{1}{\sqrt{2}}(1,0,0,1),
$$

$P$ and $S$ are the nucleon momentum and spin, $S_{T}^{\mu}$ is the transverse-spin vector $[25,32], \xi$ is the space-time coordinate, the variable $x$ is the momentum fraction carried by a parton and it is defined by $k^{+}=x P^{+}$, and the lightcone variables $a^{ \pm}$indicate $a^{ \pm}=\left(a^{0} \pm a^{3}\right) / \sqrt{2}$. In eq. (2.1), the gauge link is abbreviated. In this paper, the momentum and renormalization scale dependence $\left(Q^{2}, \mu^{2}\right)$ is not explicitly written in the PDFs and structure functions.

The moments of eq. (2.1) become

$$
\begin{aligned}
& \frac{1}{2 M_{N}\left(P^{+}\right)^{n-1}} n_{\mu_{1}} \cdots n_{\mu_{n-1}}\left\langle P, S\left|R^{\sigma\left\{\mu_{1} \cdots \mu_{n-1}\right\}}\right| P, S\right\rangle \\
& =\bar{n}^{\sigma}(S \cdot n) \int_{-1}^{1} d x x^{n-1} g_{1 L}(x)+S_{T}^{\sigma} \int_{-1}^{1} d x x^{n-1} g_{T}(x),
\end{aligned}
$$

by keeping the terms up to twist 3. Hereafter, the twist- 4 distribution function $g_{3 L}$ and twist- 4 terms are neglected in this section. The structure functions are classified by the twist, which is defined by the mass dimension minus spin, in the operator product expansion [32-38]. The local operators $R^{\sigma\left\{\mu_{1} \cdots \mu_{n-1}\right\}}$ for describing the polarized structure functions are defined by

$$
R^{\sigma\left\{\mu_{1} \cdots \mu_{n-1}\right\}}=i^{n-1} \bar{\psi} \gamma^{\sigma} \gamma_{5} D^{\left\{\mu_{1}\right.} \cdots D^{\left.\mu_{n-1}\right\}} \psi,
$$

where $D^{\mu}$ is the QCD covariant derivative given by $D^{\mu}=\partial^{\mu}-i g A^{\mu}$ with the QCD coupling constant $g$ and the gluon field $A^{\mu}$, and the curly bracket \{\} indicates the symmetrization 
of all the Lorentz indices as defined in eq. (2.5). The higher-twist $(\geq 4)$ trace terms $g_{\mu_{i} \mu_{j}} R^{\sigma\left\{\mu_{1} \cdots \mu_{n-1}\right\}}$ and $g_{\sigma \mu_{i}} R^{\sigma\left\{\mu_{1} \cdots \mu_{n-1}\right\}}$ should be subtracted to make the operator traeless; however, they are not explicitly written in eq. (2.4). The gluon field $A^{\mu}$ contains the $\mathrm{SU}(3)$ generator $t^{a}$, defined by the Gell-Mann matrix $\lambda^{a}$ as $t^{a}=\lambda^{a} / 2$ with the color index $a$, as $A^{\mu}=A_{a}^{\mu} t^{a}$. Since these operators contain both twist-2 and twist-3 components, they should be separated so as to have definite twists as [32-35, 39, 40]

$$
\begin{aligned}
R^{\sigma\left\{\mu_{1} \cdots \mu_{n-1}\right\}} & =R^{\left\{\sigma \mu_{1} \cdots \mu_{n-1}\right\}}+R^{\left[\sigma\left\{\mu_{1}\right] \cdots \mu_{n-1}\right\}} \\
R^{\left\{\sigma \mu_{1} \cdots \mu_{n-1}\right\}} & =\frac{1}{n}\left[R^{\sigma\left\{\mu_{1} \mu_{2} \cdots \mu_{n-1}\right\}}+R^{\mu_{1}\left\{\sigma \mu_{2} \cdots \mu_{n-1}\right\}}+R^{\mu_{2}\left\{\mu_{1} \sigma \cdots \mu_{n-1}\right\}}+\cdots\right] \\
R^{\left[\sigma\left\{\mu_{1}\right] \cdots \mu_{n-1}\right\}} & =\frac{1}{n}\left[(n-1) R^{\sigma\left\{\mu_{1} \mu_{2} \cdots \mu_{n-1}\right\}}-R^{\mu_{1}\left\{\sigma \mu_{2} \cdots \mu_{n-1}\right\}}-R^{\mu_{2}\left\{\mu_{1} \sigma \cdots \mu_{n-1}\right\}}-\cdots\right]
\end{aligned}
$$

where the first term $R^{\left\{\sigma \mu_{1} \cdots \mu_{n-1}\right\}}$ has spin $n$ and the second one $R^{\left[\sigma\left\{\mu_{1}\right] \cdots \mu_{n-1}\right\}}$ has $n-1$ so that they are definite twist- 2 and twist- 3 operators. The twist- 3 operator $R^{\left[\sigma\left\{\mu_{1}\right] \cdots \mu_{n-1}\right\}}$ is obtained by expanding the nonlocal operator in the Taylor series as

$$
\xi_{\mu} \bar{\psi}(0)\left(\partial^{\mu} \gamma^{\sigma}-\partial^{\sigma} \gamma^{\mu}\right) \gamma_{5} \psi(\xi) \Longrightarrow \text { twist-3: } R^{\left[\sigma\left\{\mu_{1}\right] \cdots \mu_{n-1}\right\}},
$$

where $\partial^{\mu}=\partial / \partial \xi_{\mu}$. Then, it is written by the following operators with the gluon field tensor $G^{\mu \nu}$, the quark mass $\left(m_{q}\right)$, and equation of motion as [32]

$$
\begin{aligned}
\xi_{\mu} \bar{\psi}(0)\left(\partial^{\mu} \gamma^{\sigma}-\partial^{\sigma} \gamma^{\mu}\right) \gamma_{5} \psi(\xi)= & -g \int_{0}^{1} d t \bar{\psi}(0)\left\{i \gamma_{5}\left(t-\frac{1}{2}\right) G^{\sigma \rho}(t \xi)-\frac{1}{2} \tilde{G}^{\sigma \rho}(t \xi)\right\} \xi_{\rho} \sharp \psi(\xi) \\
& +2 m_{q} \bar{\psi}(0) \gamma_{5} \sigma^{\sigma \rho} \xi_{\rho} \psi(\xi) \\
& +\bar{\psi}(0) \gamma_{5} \sigma^{\sigma \rho} \xi_{\rho}\left(i \not D-m_{q}\right) \psi(\xi)-\bar{\psi}(0)\left(i \overleftarrow{D D}+m_{q}\right) \gamma_{5} \sigma^{\sigma \rho} \xi_{\rho} \psi(\xi)
\end{aligned}
$$

where the dual field tensor $\tilde{G}^{\mu \nu}$ is defined by $\tilde{G}^{\mu \nu}=\epsilon^{\mu \nu \rho \sigma} G_{\rho \sigma} / 2$ [41] with the convention $\epsilon^{0123}=+1$. The first line of the right-hand side is from the quark-gluon-quark correlation, the second one is the quark-mass term, and the third one is the equation-of-motion term. Scale evolution was studied by using these three types of operators; however, we do not step into such details. Interested readers may read, for example, the summary article of ref. [32].

In this way, the matrix elements of these operators are generally expressed for the nucleons as

$$
\begin{aligned}
&\left\langle P, S\left|R^{\left\{\sigma \mu_{1} \cdots \mu_{n-1}\right\}}\right| P, S\right\rangle=\frac{2}{n} a_{n} M_{N}\left[S^{\sigma} P^{\mu_{1}} \cdots P^{\mu_{n-1}}+S^{\mu_{1}} P^{\sigma} \cdots P^{\mu_{n-1}} \cdots\right] \\
&\left\langle P, S\left|R^{\left[\sigma\left\{\mu_{1}\right] \cdots \mu_{n-1}\right\}}\right| P, S\right\rangle= \frac{2}{n} d_{n} M_{N}\left[\left(S^{\sigma} P^{\mu_{1}}-S^{\mu_{1}} P^{\sigma}\right) P^{\mu_{2}} \cdots P^{\mu_{n-1}}\right. \\
&\left.+\left(S^{\sigma} P^{\mu_{2}}-S^{\mu_{2}} P^{\sigma}\right) P^{\mu_{1}} \cdots P^{\mu_{n-1}}+\cdots\right]
\end{aligned}
$$

where $a_{n}$ and $d_{n}$ are constants to indicate the magnitudes of the twist-2 and twist-3 matrix elements. The polarized structure functions $g_{1}$ and $g_{2}$ are given by $g_{1 L}$ and $g_{T}$ as $g_{1}(x)=$ $\left[g_{1 L}(x)+g_{1 L}(-x)\right] / 2$ and $g_{1}(x)+g_{2}(x)=\left[g_{T}(x)+g_{T}(-x)\right] / 2$. From eqs. (2.3), (2.4), (2.5), 
and (2.8), we obtain the moments as

$$
\begin{aligned}
\int_{0}^{1} d x x^{n-1} g_{1}(x) & =\frac{1}{2} a_{n} \\
\int_{0}^{1} d x x^{n-1}\left[g_{1}(x)+g_{2}(x)\right] & =\frac{1}{2}\left(\frac{1}{n} a_{n}+\frac{n-1}{n} d_{n}\right) .
\end{aligned}
$$

In these equations, we use $g_{1}$ and $g_{2}$ for a single flavor. The second equation is written by using the first one as

$$
\int_{0}^{1} d x x^{n-1} g_{2}(x)=\int_{0}^{1} d x x^{n-1}\left[-g_{1}(x)+\int_{x}^{1} \frac{d y}{y} g_{1}(y)\right]+\frac{n-1}{2 n} d_{n} .
$$

From this equation, the structure function $g_{2}$ is written in terms of twist-2 and twist-3 parts separately as

$$
\begin{aligned}
g_{2}(x) & =g_{2}^{W W}(x)+\bar{g}_{2}(x), \\
g_{2}^{W W}(x) & =-g_{1}(x)+\int_{x}^{1} \frac{d y}{y} g_{1}(y), \\
\int_{0}^{1} d x x^{n-1} \bar{g}_{2}(x) & =\frac{n-1}{2 n} d_{n} .
\end{aligned}
$$

Equation (2.12) is the WW relation which is valid in the twist-2 level by neglecting highertwist effects. There is also a similar relation for the chiral-odd twist-3 structure function $h_{L}$ and the twist-2 one $h_{1}$ [42-45]. In addition, higher-twist terms are explicitly written by multiparton distribution functions $[44,45]$. If the WW relation of eq. (2.12) is integrated over $x$, it becomes

$$
\int_{0}^{1} d x g_{2}^{W W}(x)=0
$$

This relation is the BC sum rule, which was originally derived by using the dispersion relation for the virtual Compton amplitude. Here, the convergence of this sum could depend on the $x$-dependent functional form of $g_{2}$ at small $x$. Furthermore, no operator is defined for $n=1$ in eq. (2.4), so that the WW relation and $\mathrm{BC}$ sum rule could not be rigorously proven in the operator-product-expansion formalism [33-36, 46-49]. On the other hand, these relations are satisfied even if perturbative QCD corrections are included in coefficient functions [35].

\section{Parton distribution functions of spin-1 hadrons and their twist-2 relation and sum rule}

For studying structure functions, sum rules provide useful information on their $x$-dependent functional forms. There are sum rules for the structure functions and TMDs of spin1 hadrons $[3,4,25]$. If tensor-polarized antiquark distributions vanish, there is a sum rule for the twist-2 collinear structure function as $\int d x b_{1}(x)=0$. In addition, due to the time-reversal invariance of collinear parton distributions, there exist the sum rules 
$\int d^{2} k_{T} h_{1 L T}\left(x, k_{T}^{2}\right)=\int d^{2} k_{T} g_{L T}\left(x, k_{T}^{2}\right)=\int d^{2} k_{T} h_{L L}\left(x, k_{T}^{2}\right)=\int d^{2} k_{T} h_{3 L L}\left(x, k_{T}^{2}\right)=0$ for the TMDs of tensor-polarized spin-1 hadrons. In this subsection, we show the existence of a new sum rule and a twist-2 relation, which are analogous to the BC sum rule and the WW relation, respectively.

\subsection{Matrix elements of nonlocal operators and parton distribution functions}

The PDFs of hadrons are often discussed by correlation functions. The PDFs of spin- $1 / 2$ nucleons are now theoretically investigated including higher-twist ones. On the other hand, the PDFs of spin-1 hadrons are not well studied especially for the tensor-polarized part. The TMDs and PDFs of spin-1 hadrons were investigated for the twist-2 in refs. [26-29], and twist-3 and twist-4 functions were recently proposed in ref. [25]. The PDFs of hadrons are generally defined from the correlation function

$$
\Phi_{i j}^{[c]}(k, P, T \mid n)=\int \frac{d^{4} \xi}{(2 \pi)^{4}} e^{i k \cdot \xi}\left\langle P, T\left|\bar{\psi}_{j}(0) W^{[c]}(0, \xi) \psi_{i}(\xi)\right| P, T\right\rangle,
$$

which is related to the amplitude to extract a parton from a hadron and then to insert it into the hadron at a different spacetime point $\xi$. Here, $k$ is the quark momentum, the hadron momentum and tensor polarization are denoted by $P$ and $T$, respectively, $W^{[c]}$ is the gauge link for satisfying the color gauge invariance, and $c$ indicates the integral path. We do not write the spin vector polarization $S$ explicitly in eq. (3.1) because only the tensor polarization $T$, which is specific to hadrons with spin $\geq 1$, is investigated in this paper. The vector polarization part is essentially the same as the one for the spin- $1 / 2$ nucleons. From the general correlation function in eq. (3.1), we obtain the collinear correlation function by integrating it over the lightcone momentum $k^{-}$and the transverse momentum $k_{T}$, and fixing the $k^{+}$component as

$$
\begin{aligned}
\Phi_{i j}(x, P, T) & =\int d^{2} k_{T} d k^{+} d k^{-} \Phi_{i j}^{[c]}(k, P, T \mid n) \delta\left(k^{+}-x P^{+}\right), \\
& =\int \frac{d \xi^{-}}{2 \pi} e^{i x P^{+} \xi^{-}}\left\langle P, T\left|\bar{\psi}_{j}(0) W(0, \xi \mid n) \psi_{i}(\xi)\right| P, T\right\rangle_{\xi^{+}=0, \vec{\xi}_{T}=0} .
\end{aligned}
$$

Here, $W(a, b \mid n)$ indicates the gauge line connecting $a=\left(a^{+}, a^{-}, \vec{a}_{T}\right)$ to $b=\left(b^{+}, b^{-}, \vec{b}_{T}\right)$ along the straight lightcone direction of $\xi^{-}$. Since the link is along the straight line, there is no path- $c$ dependence.

The TMDs and collinear PDFs for spin-1 hadrons are defined in various traces of the TMD and collinear correlation function as $\operatorname{Tr}(\Phi \Gamma)$, where $\Gamma$ is expressed by $\gamma$ matrices, in ref. [25]. In this subsection, the tensor-polarized collinear PDFs are studied and the gauge link $W(0, \xi \mid n)$ is not explicitly written. Later, the Fock-Schwinger gauge $\xi_{\mu} A^{\mu}(\xi)=0$ [50] is used from section 3.2 and the gauge link is unity $W(0, \xi \mid n)=1$ in any case for the collinear PDFs. However, this link appears in section 3.2, for example in eq. (3.20), for obtaining our WW- and BC-like relations because its total derivative does not vanish. 
Here, we are interested in collinear PDFs which are defined in the trace as

$$
\begin{aligned}
\Phi^{[\Gamma]}(x, P, T) & \equiv \frac{1}{2} \operatorname{Tr}[\Phi(x, P, T) \Gamma]=\frac{1}{2} \Phi_{i j}(x, P, T)(\Gamma)_{j i} \\
& =\frac{1}{2} \int \frac{d \xi^{-}}{2 \pi} e^{i x P^{+} \xi^{-}}\langle P, T|\bar{\psi}(0) \Gamma \psi(\xi)| P, T\rangle_{\xi^{+}=0, \vec{\xi}_{T}=0}
\end{aligned}
$$

The tensor polarization $T^{\mu \nu}$ is generally expressed by the polarizations $S_{L L}, S_{L T}^{\mu}$, and $S_{T T}^{\mu \nu}$ as $[17,18,25]$

$$
\begin{aligned}
T^{\mu \nu}=\frac{1}{2}[ & \frac{4}{3} S_{L L} \frac{\left(P^{+}\right)^{2}}{M^{2}} \bar{n}^{\mu} \bar{n}^{\nu}-\frac{2}{3} S_{L L}\left(\bar{n}^{\{\mu} n^{\nu\}}-g_{T}^{\mu \nu}\right)+\frac{1}{3} S_{L L} \frac{M^{2}}{\left(P^{+}\right)^{2}} n^{\mu} n^{\nu} \\
& \left.+\frac{P^{+}}{M} \bar{n}^{\{\mu} S_{L T}^{\nu\}}-\frac{M}{2 P^{+}} n^{\{\mu} S_{L T}^{\nu\}}+S_{T T}^{\mu \nu}\right]
\end{aligned}
$$

where $a^{\{\mu} b^{\nu\}}$ is the symmetrized combination $a^{\{\mu} b^{\nu\}}=a^{\mu} b^{\nu}+a^{\nu} b^{\mu}$. The tensor-polarization parameters $S_{L L}, S_{L T}^{\mu}$, and $S_{T T}^{\mu \nu}$ are explained in appendix of refs. [26-29]. The $S_{L L}$ is associated with the tensor polarization along the $z$ direction, $S_{T T}^{\mu \nu}$ is the linear polarization in the transverse plane, and $S_{L T}^{\mu}$ is the polarization in the plane in-between.

We investigate possible relations of tensor-polarized PDFs $f_{1 L L}$ and $f_{L T}$, where the function $f_{1 L L}$ is twist 2 and $f_{L T}$ is twist 3 , in analogy to the WW and BC relations for $g_{1}$ and $g_{2}$. The function $f_{1 L L}$ is defined in $\Phi^{\left[\gamma^{+}\right]}(x, P, T)$, and $f_{L T}$ is in $\Phi^{\left[\gamma^{i}\right]}(x, P, T)$, and the actual expressions are given in the TMD form in ref. [25]. The twist-4 function $f_{3 L L}$ exists in another vector type correlation function $\Phi^{\left[\gamma^{-}\right]}(x, P, T)$, so that it is also included in the following discussion of this subsection. There is another twist-3 function $e_{L L}$ defined in $\Phi^{[\mathbf{1}]}(x, P, T)$, where $\mathbf{1}$ is the $4 \times 4$ identity matrix, and it is also listed in this subsection. There are related works on the TMDs and PDFs of spin- 1 hadrons $[2,11,26-$ 29]. Integrating the TMD expressions of eqs. (33), (43), and (52) in ref. [25] for $\Gamma=\gamma^{+}$, $\gamma^{i}, \gamma^{-}$, and 1 over the transverse momentum $\vec{k}_{T}$, we obtain PDF expressions in terms of operator matrix elements as

$$
\begin{aligned}
\Phi^{\left[\gamma^{+}\right]}(x, P, T) & =\int \frac{d \xi^{-}}{4 \pi} e^{i x P^{+} \xi^{-}}\left\langle P, T\left|\bar{\psi}(0) \gamma^{+} \psi(\xi)\right| P, T\right\rangle_{\xi^{+}=0, \vec{\xi}_{T}=0}=S_{L L} f_{1 L L}(x), \\
\Phi^{\left[\gamma^{\alpha}\right]}(x, P, T) & =\int \frac{d \xi^{-}}{4 \pi} e^{i x P^{+} \xi^{-}}\left\langle P, T\left|\bar{\psi}(0) \gamma^{\alpha} \psi(\xi)\right| P, T\right\rangle_{\xi^{+}=0, \vec{\xi}_{T}=0}=\frac{M}{P^{+}} S_{L T}^{\alpha} f_{L T}(x), \\
\Phi^{\left[\gamma^{-}\right]}(x, P, T) & =\int \frac{d \xi^{-}}{4 \pi} e^{i x P^{+} \xi^{-}}\left\langle P, T\left|\bar{\psi}(0) \gamma^{-} \psi(\xi)\right| P, T\right\rangle_{\xi^{+}=0, \vec{\xi}_{T}=0}=\frac{M^{2}}{\left(P^{+}\right)^{2}} S_{L L} f_{3 L L}(x), \\
\Phi^{[\mathbf{1}]}(x, P, T) & =\int \frac{d \xi^{-}}{4 \pi} e^{i x P^{+} \xi^{-}}\langle P, T|\bar{\psi}(0) \psi(\xi)| P, T\rangle_{\xi^{+}=0, \vec{\xi}_{T}=0}=\frac{M}{P^{+}} S_{L L} e_{L L}(x),
\end{aligned}
$$

where $\alpha$ is the transverse index $\alpha=1$ or 2 and this transverse $\alpha$ notation is used throughout this paper. The tensor-polarized PDFs $f_{1 L L}(x), f_{L T}(x), f_{3 L L}(x)$, and $e_{L L}(x)$ are expressed by the matrix elements of the nonlocal operators with different $\gamma$ matrices in eq. (3.5). The collinear PDFs are often written from the TMDs as

$$
f(x)=\int d^{2} k_{T} f\left(x, k_{T}^{2}\right)
$$


However, we should note that this integral equation could not be a general relation due to the ultraviolet divergence in the transverse-momentum integral [51]. Futhermore, the TMDs $f_{L T}^{\prime}\left(x, k_{T}^{2}\right)$ and $f_{L T}^{\perp}\left(x, k_{T}^{2}\right)$ are combined to express them as [25] $f_{L T}\left(x, k_{T}^{2}\right) \equiv$ $f_{L T}^{\prime}\left(x, k_{T}^{2}\right)-\frac{k_{T}^{2}}{2 M^{2}} f_{L T}^{\perp}\left(x, k_{T}^{2}\right)$. Therefore, two functions among $f_{L T}, f_{L T}^{\prime}$, and $f_{L T}^{\perp}$ are independent, and $f_{L T}$ and $f_{L T}^{\perp}$ are selected in the table IV of ref. [25]. In eq. (3.5), $M$ is the spin-1 hadron mass, $f_{1 L L}, f_{L T}$, and $f_{4 L L}$ are twist-2, twist-3, and twist-4 distribution functions, respectively. In this way, the collinear correlation function is written up to twist-4 as

$$
\Phi(x, P, T)=\frac{1}{2}\left[S_{L L} \hbar f_{1 L L}(x)+\frac{M}{P^{+}} S_{L L} e_{L L}(x)+\frac{M}{P^{+}} \$_{L T} f_{L T}(x)+\frac{M^{2}}{\left(P^{+}\right)^{2}} S_{L L} \not h f_{3 L L}(x)\right] .
$$

Then, the matrix element of eq. (3.3) is expressed by the Fourier transform of $\Phi^{\left[\gamma^{\mu}\right]}(x, P, T)$ expressed by the PDFs as

$$
\begin{aligned}
& \left\langle P, T\left|\bar{\psi}(0) \gamma^{\mu} \psi(\xi)\right| P, T\right\rangle_{\xi^{+}=0, \vec{\xi}_{T}=0} \\
& =\int_{-1}^{1} d x e^{-i x P^{+} \xi^{-}} 2 P^{+}\left[S_{L L} \bar{n}^{\mu} f_{1 L L}(x)+\frac{M}{P^{+}} S_{L T}^{\mu} f_{L T}(x)+\frac{M^{2}}{\left(P^{+}\right)^{2}} S_{L L} n^{\mu} f_{3 L L}(x)\right] .
\end{aligned}
$$

Therefore, the matrix element of the vector operator is given by the three collinear PDFs $f_{1 L L}(x), f_{L T}(x)$, and $f_{3 L L}(x)$ for the tensor-polarized hadron. In particular, we derive a useful relation and a sum rule for the twist-3 functions $f_{L T}(x)$ in this work.

Since antiquark distribution are discussed later in deriving twist-2 relations, we briefly explain them. We define the collinear antiquark correlation function in the same way as $[17,18,52,53]$

$$
\begin{aligned}
\bar{\Phi}_{i j}(x, P, T) & =-\int \frac{d \xi^{-}}{2 \pi} e^{-i x P^{+} \xi^{-}}\left\langle P, T\left|\bar{\psi}_{j}(0) W(0, \xi \mid n) \psi_{i}(\xi)\right| P, T\right\rangle_{\xi^{+}=0, \vec{\xi}_{T}=0} \\
& =-\Phi_{i j}(-x, P, T) .
\end{aligned}
$$

Therefore, the antiquark correlation function is related to the quark correlation function at negative $x$, so that the antiquark distributions are described by the quark distributions at negative $x, q(x<0)$. On the other hand, the charge-conjugate correlation function, in which the antiquark distributions $\bar{q}(x)$ are defined, is given by the conjugate spinor $\psi^{C} \equiv C \bar{\psi}^{T}$ with $C=i \gamma^{2} \gamma^{0}$ and $A_{\mu}^{C}=-A_{\mu}$ as

$$
\Phi_{i j}^{C}(x, P, T)=\int \frac{d \xi^{-}}{2 \pi} e^{i x P^{+} \xi^{-}}\left\langle P, T\left|\bar{\psi}_{j}^{C}(0) W^{C}(0, \xi \mid n) \psi_{i}^{C}(\xi)\right| P, T\right\rangle_{\xi^{+}=0, \vec{\xi}_{T}=0} .
$$

These equations indicate the relation between them as

$$
\Phi^{C}(x, P, T)=-C[\bar{\Phi}(x, P, T)]^{\mathscr{T}} C^{\dagger},
$$

where $\mathscr{T}$ indicates the transposed matrix. The antiquark distributions $\bar{q}(x)$ are then defined by using this conjugate correlation function. The relations between the various antiquark 
distributions $\bar{q}(x)$ and the corresponding "quark" distributions $q(x<0)$ are explicitly written in the end of section 3.1. Next, we show relations between the antiquark distributions and the quark ones at negative $x$. The relation between the correlation function $\bar{\Phi}$ and the conjugate one $\Phi^{C}$ is

$$
\Phi^{C[\Gamma]}(x, P, T)=\left\{\begin{array}{ll}
+\bar{\Phi}^{[\Gamma]}(x, P, T)=-\Phi^{[\Gamma]}(-x, P, T) & \text { for } \Gamma=\gamma^{\mu}, \sigma^{\mu \nu}, i \gamma_{5} \sigma^{\mu \nu} \\
-\bar{\Phi}^{[\Gamma]}(x, P, T)=+\Phi^{[\Gamma]}(-x, P, T) & \text { for } \Gamma=\mathbf{1}, i \gamma_{5}, \gamma_{5} \gamma^{\mu}
\end{array},\right.
$$

from eqs. (3.9), (3.10), (3.11), and (3.3). By taking $\Gamma=\gamma^{\mu}$ or 1 in eqs. (3.7) and (3.12) the antiquark distributions are related to the quark distributions as

$$
\begin{aligned}
\bar{f}_{1 L L}(x) & =-f_{1 L L}(-x), \quad \bar{f}_{L T}(x)=-f_{L T}(-x), \quad \bar{f}_{3 L L}(x)=-f_{3 L L}(-x), \\
\bar{e}_{L L}(x) & =e_{L L}(-x),
\end{aligned}
$$

where the sign is opposite for the chiral-odd distribution function $e_{L L}(x)$.

Let us consider the matrix element of non-local vector operator $\bar{\psi}(0) \gamma^{\mu} \psi(\xi)$ in the region which is not necessarily on the lightcone, where eq. (3.8) was obtained, for calculating the matrix element with its derivative. The Fourier transform of the vector-operator matrix element is the correlation function, which is expanded by the linear terms of the tensor polarization $T^{\mu \nu}$ as given in eq. (20) of ref. [25]. Therefore, the matrix element should be generally expressed in terms of three terms $(\xi \cdot T \cdot \xi) P^{\mu},(\xi \cdot T \cdot \xi) \xi^{\mu}$, and $T^{\mu \nu} \xi_{\nu}$ which are linear in the tensor polarization with the available Lorentz vectors $P^{\mu}, \xi^{\mu}$, and $T^{\mu \nu} \xi_{\nu}$ as

$$
\begin{aligned}
\langle P, T| & \bar{\psi}(0) \gamma^{\mu} \psi(\xi)|P, T\rangle \\
& =\int_{-1}^{1} d x e^{-i x P \cdot \xi}\left[\xi \cdot T \cdot \xi\left\{A(x) P^{\mu}+B(x) \xi^{\mu}\right\}+C(x) T^{\mu \nu} \xi_{\nu}\right],
\end{aligned}
$$

where $\xi \cdot T \cdot \xi$ is defined by $\xi \cdot T \cdot \xi=\xi_{\mu} T^{\mu \nu} \xi_{\nu}$ and $\xi$ may not be on the lightcone. The term $T^{\mu \nu} P_{\nu}$ does not exist in eq. (3.14) because it vanishes identically $T^{\mu \nu} P_{\nu}=0$. Here, $A(x), B(x)$, and $C(x)$ are coefficients to be determined. In this expansion, twist- 4 effects are neglected, so that the twist- 4 function $f_{3 L L}(x)$ does not appear in the following discussions. The tensor polarization $T^{\mu \nu}$ contains the three types of polarizations $S_{L L}, S_{L T}^{\mu}$, and $S_{T T}^{\mu \nu}$, and the right-hand side of eq. (3.14) should be expressed by them. We find the factors $A(x), B(x)$, and $C(x)$ so that eq. (3.14) becomes eq. (3.8) in the lightcone limit $\xi^{2} \rightarrow 0$ $\left(\xi^{\mu}=\xi^{-} n^{\mu}, \xi^{+}=\vec{\xi}_{T}=0\right)$. In this limit, the factors $\xi \cdot T \cdot \xi$ and $T^{\mu \nu} \xi_{\nu}$ are expressed by the tensor polarization factors $S_{L L}$ and $S_{L T}^{\mu}$ by using eq. (3.4) as

$$
\begin{aligned}
\xi \cdot T \cdot \xi & =\frac{2}{3 M^{2}}\left(P^{+} \xi^{-}\right)^{2} S_{L L}, \\
T^{\mu \nu} \xi_{\nu} & =\frac{2}{3 M^{2}}\left[\left(P^{+}\right)^{2} \xi^{-} \bar{n}^{\mu}-\frac{1}{2} M^{2} \xi^{-} n^{\mu}\right] S_{L L}+\frac{1}{2 M} P^{+} \xi^{-} S_{L T}^{\mu}
\end{aligned}
$$

Then, $A(x), B(x)$, and $C(x)$ are obtained as

$$
\begin{aligned}
& A(x)=\frac{3 M^{2}}{(P \cdot \xi)^{2}}\left[f_{1 L L}(x)-\frac{4}{3} f_{L T}(x)\right], B(x)=\frac{3 M^{4}}{2(P \cdot \xi)^{3}}\left[-f_{1 L L}(x)+\frac{8}{3} f_{L T}(x)\right] \\
& C(x)=\frac{4 M^{2}}{P \cdot \xi} f_{L T}(x)
\end{aligned}
$$


in the lightcone limit with $P \cdot \xi=P^{+} \xi^{-}$. In order to derive a twist-2 relation and a sum rule, we need to investigate the twist-3 matrix element $\xi_{\mu}\left\langle P, T\left|\bar{\psi}(0)\left(\partial^{\mu} \gamma^{\alpha}-\partial^{\alpha} \gamma^{\mu}\right) \psi(\xi)\right| P, T\right\rangle$ as the derivative operator is given in eqs. (2.6) and (2.7) for $g_{2}$. Taking the transverse index $\alpha=1$ or 2 and considering the lightcone limit, we obtain the relation

$$
\begin{aligned}
& \xi_{\mu}\left\langle P, T\left|\bar{\psi}(0)\left(\partial^{\mu} \gamma^{\alpha}-\partial^{\alpha} \gamma^{\mu}\right) \psi(\xi)\right| P, T\right\rangle \\
& =2 M S_{L T}^{\alpha} \int_{-1}^{1} d x e^{-i x P^{+} \xi^{-}}\left[-\frac{3}{2} f_{1 L L}(x)+f_{L T}(x)-\frac{d}{d x}\left\{x f_{L T}(x)\right\}\right],
\end{aligned}
$$

from eq. (3.14). In this way, it becomes possible to identify the twist-3 part of the function $f_{L T}(x)$ in connection with the twist-2 function $f_{1 L L}(x)$.

Since the nonlocal operator of the left-hand side in eq. (3.17) gives rise to the twist-3 operators as explained in section 2, the right-hand side should vanish if higher-twist effects are neglected. It leads to the $\mathrm{WW}$ - and BC-like twist-2 relations for $f_{L T}$ and $f_{1 L L}$. However, we investigate further in this work by defining possible multiparton distribution functions for the tensor-polarized spin-1 hadron, and then we explicitly show that the left-hand side is expressed by these multiparton distribution functions. Namely, we try to obtain the full decomposition of $f_{L T}$ into the twist-2 and twist-3 terms.

\subsection{Twist-3 matrix element and multiparton distribution functions}

For specifying twist-3 effects, we derived the expression of twist-3 terms in eq. (3.17) in terms of the tensor-polarized distribution functions. In general, the twist-3 terms are described by multiparton (three-parton in this work) distribution functions [32, 50, 54]. In order to derive a twist- 2 relation and a sum rule for the tensor-polarized PDFs, we try to connect the derivative terms in the left-hand side of eq. (3.17) to the multiparton distribution functions. For this purpose, we try to express the derivative terms by the nonlocal quark-gluon operators in this subsection.

The Fock-Schwinger gauge $x_{\mu} A^{\mu}(x)=0$ is used in our formalism, so that the gluon field is expressed by the field strength tensor by introducing the variable $t$ as

$$
A^{\nu}(\xi)=\int_{0}^{1} d t t \xi_{\mu} G^{\mu \nu}(t \xi)
$$

where $G^{\mu \nu}=\partial^{\mu} A^{\nu}-\partial^{\nu} A^{\mu}-i g\left[A^{\mu}, A^{\nu}\right]$. The gauge link is generally expressed as

$$
W(0, \xi)=\mathcal{P} \exp \left[-i g \int_{0}^{1} d t \xi_{\mu} A^{\mu}(t \xi)\right]
$$

where the integral path is the direct one from 0 to $\xi$. If the Fock-Schwinger gauge is taken, the gauge link becomes unity. However, the total derivative $\bar{\partial}^{\alpha} W$ does not vanish and it is used for relating the derivative relation of eq. (3.17) to the field tensor and subsequently to the multiparton distribution functions. We consider that the total derivative $\left.\frac{\partial}{\partial\left(\Delta \xi^{\rho}\right)} W(\Delta \xi, \xi+\Delta \xi)\right|_{\Delta \xi \rightarrow 0}$, which is given by the field tensor $G^{\rho \mu}$ and the gluon field $A^{\rho}$ as

$$
\left.\bar{\partial}_{\rho} W(0, \xi) \equiv \frac{\partial}{\partial\left(\Delta \xi^{\rho}\right)} W(\Delta \xi, \xi+\Delta \xi)\right|_{\Delta \xi \rightarrow 0}=-i g \int_{0}^{1} d t \xi^{\nu} G_{\rho \nu}(t \xi)-i g\left[A_{\rho}(\xi)-A_{\rho}(0)\right] .
$$


From eq. (3.20), the derivative of the local operator $\bar{\psi}(0) W \Gamma \psi(\xi)$ becomes

$$
\bar{\partial}_{\rho} \bar{\psi}(0) W(0, \xi) \Gamma \psi(\xi)=\bar{\psi}(0)\left(\overleftarrow{D}_{\rho}+\vec{D}_{\rho}\right) \Gamma \psi(\xi)-i g \int_{0}^{1} d t \xi^{\nu} G_{\rho \nu}(t \xi) \bar{\psi}(0) \Gamma \psi(\xi)
$$

where the covariant derivatives are given by $\overleftarrow{D}_{\rho}=\overleftarrow{\partial}_{\rho}+i g A_{\rho}$ and $\vec{D}_{\rho}=\vec{\partial}_{\rho}-i g A_{\rho}$

Next, we express the left-hand side of eq. (3.17) in terms of the covariant derivatives and the field tensor as

$$
\begin{aligned}
\bar{\psi}(0)\left(\partial^{\mu} \gamma^{\alpha}-\partial^{\alpha} \gamma^{\mu}\right) \psi(\xi)= & \bar{\psi}(0)\left(\vec{D}^{\mu} \gamma^{\alpha}-\vec{D}^{\alpha} \gamma^{\mu}\right) \psi(\xi) \\
& -\bar{\psi}(0) \gamma^{\mu} \psi(\xi) i g \int_{0}^{1} d t t \xi_{\rho} G^{\rho \alpha}(t \xi) .
\end{aligned}
$$

The index $\alpha$ is the transverse one $(\alpha=1,2)$; however, all the following equations within this subsection are valid as a general 4-dimensional Lorentz index. By the identity

$$
\gamma^{\rho} \sigma^{\alpha \mu}=i\left(g^{\alpha \rho} \gamma^{\mu}-g^{\mu \rho} \gamma^{\alpha}\right)-\epsilon^{\alpha \mu \rho \sigma} \gamma_{\sigma} \gamma_{5}
$$

the covariant derivative term becomes $\vec{D}^{\mu} \gamma^{\alpha}-\vec{D}^{\alpha} \gamma^{\mu}=i\left(\vec{D} \sigma^{\alpha \mu}-\sigma^{\alpha \mu} \overrightarrow{\not D}\right) / 2$. Then, using eq. (3.21) with the $\Gamma$ factor $\Gamma=\gamma^{\rho} \sigma^{\alpha \mu}$, we obtain the first derivative terms in the right-hand side of eq. (3.22) as

$$
\begin{aligned}
\bar{\psi}(0)\left(\vec{D}^{\mu} \gamma^{\alpha}-\right. & \left.\vec{D}^{\alpha} \gamma^{\mu}\right) \psi(\xi)=-\frac{i}{2} \bar{\psi}(0)\left(\sigma^{\alpha \mu} \overrightarrow{D D}+\overleftarrow{\not D} \sigma^{\alpha \mu}\right) \psi(\xi) \\
& -\frac{g}{2} \int_{0}^{1} d t \xi_{\nu} G^{\rho \nu}(t \xi) \bar{\psi}(0) \gamma_{\rho} \sigma^{\alpha \mu} \psi(\xi)+\frac{i}{2} \bar{\partial}_{\rho}\left\{\bar{\psi}(0) \gamma^{\rho} \sigma^{\alpha \mu} \psi(\xi)\right\}
\end{aligned}
$$

The third term of the right-hand side $\left(\gamma_{\rho} \sigma^{\alpha \mu}\right)$ is written by the antisymmetric tensor $\epsilon^{\alpha \mu \rho \sigma}$ of eq. (3.23), and then it is given by the dual field tensor $\widetilde{G}^{\mu \nu}$ as

$$
\xi_{\mu} \xi_{\nu} G_{\rho \tau}(t \xi) g^{\nu \tau} \epsilon^{\alpha \mu \rho \sigma}=2 \xi_{\mu} \xi^{\alpha} \widetilde{G}^{\mu \sigma}-2 \xi^{2} \widetilde{G}^{\alpha \sigma}+\xi_{\mu} \xi^{\tau} G_{\tau \rho} \epsilon^{\alpha \mu \rho \sigma}+2 \xi_{\mu} \xi^{\sigma} \widetilde{G}^{\alpha \mu}
$$

where the relation $g^{\nu \tau} \epsilon^{\alpha \mu \rho \sigma}=g^{\nu \alpha} \epsilon^{\tau \mu \rho \sigma}+g^{\nu \mu} \epsilon^{\alpha \tau \rho \sigma}+g^{\nu \rho} \epsilon^{\alpha \mu \tau \sigma}+g^{\nu \sigma} \epsilon^{\alpha \mu \rho \tau}$ was used. We may note that the third term in the right-hand side of eq. (3.25) is identical to the left-hand side with the minus sign, so that the factor of 2 in front of the dual field tensor is dropped for calculating the left-hand-side term. Therefore, the third term of eq. (3.24) contracted with $\xi_{\mu}$ becomes

$$
\begin{aligned}
-\frac{g}{2} \xi_{\mu} \int_{0}^{1} d t \xi^{\nu} G_{\rho \nu}(t \xi) \bar{\psi}(0) \gamma^{\rho} \sigma^{\alpha \mu} \psi(\xi)= & \frac{g}{2} \int_{0}^{1} d t \bar{\psi}(0)\left[-i \xi_{\mu} G^{\alpha \mu}(t \xi) \notin+\xi_{\mu} \widetilde{G}^{\alpha \mu}(t \xi) \notin \gamma_{5}\right. \\
& \left.-\left\{\xi^{2} \widetilde{G}^{\alpha \sigma}(t \xi)-\xi_{\mu} \xi^{\alpha} \widetilde{G}^{\mu \sigma}(t \xi)\right\} \gamma_{\sigma} \gamma_{5}\right] \psi(\xi) .
\end{aligned}
$$

Substituting eqs. (3.24) and (3.26) into eq. (3.22), we obtain

$$
\begin{aligned}
& \xi_{\mu} \bar{\psi}(0)\left(\vec{\partial}^{\mu} \gamma^{\alpha}-\vec{\partial}^{\alpha} \gamma^{\mu}\right) \psi(\xi)=g \int_{0}^{1} d t \bar{\psi}(0)\left[i\left(t-\frac{1}{2}\right) G^{\alpha \mu}(t \xi)-\frac{1}{2} \gamma_{5} \widetilde{G}^{\alpha \mu}(t \xi)\right] \xi_{\mu} \xi^{\psi} \psi(\xi) \\
& +\frac{g}{2} \int_{0}^{1} d t \bar{\psi}(0)\left[\xi_{\mu} \xi^{\alpha} \widetilde{G}^{\mu \sigma}(t \xi)-\xi^{2} \widetilde{G}^{\alpha \sigma}(t \xi)\right] \gamma_{\sigma} \gamma_{5} \psi(\xi) \\
& -\frac{i}{2} \xi_{\mu} \bar{\psi}(0) \sigma^{\alpha \mu}\left(\vec{D}-m_{q}\right) \psi(\xi)-\frac{i}{2} \xi_{\mu} \bar{\psi}(0)\left(\overleftarrow{I D}+m_{q}\right) \sigma^{\alpha \mu} \psi(\xi)+\frac{i}{2} \xi_{\mu} \bar{\partial}_{\rho}\left\{\bar{\psi}(0) \gamma^{\rho} \sigma^{\alpha \mu} \psi(\xi)\right\}
\end{aligned}
$$


where the quark-mass term like the second line of eq. (2.7) does not exist due to the operator difference. In this equation, the first term in the second line of r.h.s. is a twist-4 contribution and the next term vanishes in the lightcone limit $\xi^{2}=0$. The first two terms in the third line are the equation-of-motion terms, and the last term could be neglected since the total derivative term vanishes in the forward matrix elements [32, 50]. Therefore, at the twist-3 level, the relation is given by

$$
\xi_{\mu} \bar{\psi}(0)\left(\partial^{\mu} \gamma^{\alpha}-\partial^{\alpha} \gamma^{\mu}\right) \psi(\xi)=g \int_{0}^{1} d t \bar{\psi}(0)\left[i\left(t-\frac{1}{2}\right) G^{\alpha \mu}(t \xi)-\frac{1}{2} \gamma_{5} \widetilde{G}^{\alpha \mu}(t \xi)\right] \xi_{\mu} \xi \psi(\xi),
$$

In this way, the derivative terms in the left-hand side of eq. (3.17) are given by the field tensor $G^{\mu \nu}$ and the dual one $\tilde{G}^{\mu \nu}$.

The next step is to relate the field-tensor terms of eq. (3.28) to twist-3 multiparton (three-parton) distribution functions. For this purpose, we define a quark-gluon-quark correlation function $\Phi_{G}^{\mu}\left(x_{1}, x_{2}\right)$ for a tensor-polarized spin-1 hadron in terms of the field tensor $G^{\mu \nu}$. For defining the correlation function, only the transverse component of the gluon field is considered in the lightcone formalism as the leading term [40, 52, 53, 55]. We have been using the Fock-Schwinger gauge $(\xi \cdot A=0)$ in this paper; however, it is identical to the lightcone gauge $(n \cdot A=0)$ in the lightcone limit because of the relation $\xi \cdot A=\xi^{-} n \cdot A=\xi^{-} A^{+}=0$. The multiparton (quark-gluon-quark) correlation function is defined by using the transverse gluon field $A^{\alpha}\left(=A_{T}^{\alpha}\right)$, where $\alpha$ is taken as the transverse index $\alpha=1$ or 2 . In the lightcone gauge $A^{+}=0$, the field tensor is expressed by the gluon field as $G^{+\alpha}=\partial^{+} A^{\alpha}$, so that the correlation function is defined with $G^{+\alpha}$ as

$$
\left(\Phi_{G}^{\alpha}\right)_{i j}\left(x_{1}, x_{2}\right)=\int \frac{d \xi_{1}^{-}}{2 \pi} \frac{d \xi_{2}^{-}}{2 \pi} e^{i x_{1} P^{+} \xi_{1}^{-}} e^{i\left(x_{2}-x_{1}\right) P^{+} \xi_{2}^{-}}\left\langle P, T\left|\bar{\psi}_{j}(0) g G^{+\alpha}\left(\xi_{2}^{-}\right) \psi_{i}\left(\xi_{1}^{-}\right)\right| P, T\right\rangle .
$$

Due to the relation $G^{+\alpha}=\partial^{+} A^{\alpha}$, the same correlation-function expression, which is derived in the following, should be valid for the correlation function defined with the gluon field $A^{\alpha}$ instead of $G^{+\alpha}$, except for minor changes discussed after eq. (3.33). Here, the gauge link is not written because of the lightcone gauge. The conditions of Hermiticity, parity invariance, and time-reversal invariance are given in refs. [25, 52, 53].

Next, we try to express the multiparton correlation function in terms of possible Lorentz vectors with the index $\alpha$. There was some study on the multiparton correlation function for the deuteron in ref. [11]. In this work, we try to provide the full expression for the multiparton correlation function for spin- 1 hadrons, and then we relate them to the twist- 2 relation and sum rule for $f_{L T}$. Here, only the tensor-polarization is considered since the unpolarized and vector-polarization correlation functions have already investigated for the spin-1/2 nucleons. Then, the correlation function of eq. (3.29) should be proportional to the tensor polarization $T^{\mu \nu}$ as shown in eq. (20) of ref. [25]. The correlation function in eq. (3.29) has Dirac spinor indices $i$ and $j$, so that it is expressed by the Dirac $\gamma$ matrices such as $\gamma^{\mu}$ and $\gamma^{\mu} \gamma^{\nu}$. Three and more $\gamma$ types are not independent from these one- and two- $\gamma$ types because of the relation $\gamma^{\mu} \gamma^{\rho} \gamma^{\nu}=S^{\mu \rho \nu \beta} \gamma_{\beta}+i \epsilon^{\mu \rho \nu \beta} \gamma_{\beta} \gamma_{5}$ with $S^{\mu \rho \nu \beta}=g^{\mu \rho} g^{\nu \beta}+g^{\mu \beta} g^{\rho \nu}-g^{\mu \nu} g^{\rho \beta}$. 
In terms with $T^{\mu \alpha}$, the Lorentz index $\mu$ should be contracted with other vectors. We note that the hadron momentum $P^{\mu}$ is expressed by the two lightcone vectors $n^{\mu}$ and $\bar{n}^{\mu}$ $\left(P^{\mu}=P^{+} \bar{n}^{\mu}+M^{2} n^{\mu} /\left(2 P^{+}\right)\right)$, where the $n^{\mu}$ term is suppressed by the factor $O\left(M^{2} /\left(P^{+}\right)^{2}\right)$. First, let us take the contraction of $T^{\mu \alpha}$ in eq. (3.4) with the lightcone vector $n_{\mu}$ to obtain $n_{\mu} T^{\mu \alpha} \hbar=\frac{P^{+}}{2 M} S_{L T}^{\alpha} \hbar$, where $\frac{\hbar}{h}$ is multiplied for including the $\gamma$ matrix, and the higher-twist $\not h$ terms are not included. Namely, the term $n_{\mu} T^{\mu \alpha}$ is given by the tensor polarization vector $S_{L T}^{\alpha}$. The term $\bar{n}_{\mu} T^{\mu \alpha}$ is expressed as $\bar{n}_{\mu} T^{\mu \alpha}=-\frac{M^{2}}{2\left(P^{+}\right)^{2}} n_{\mu} T^{\mu \alpha}$, so that it is not an independent term. In the same way, the term $\gamma_{\mu} T^{\mu \alpha}$ is not independent from $n_{\mu} T^{\mu \alpha}$ as they are related with each other by $n_{\mu} T^{\mu \alpha} \not h=\gamma_{\mu} T^{\mu \alpha}$ in the lightcone limit. On the other hand, $\gamma_{T \mu} T^{\mu \alpha} \not h$ is an independent term. Therefore, we have two independent terms associated with $T^{\mu \alpha}$ :

$$
n_{\mu} T^{\mu \alpha} \not h=\frac{P^{+}}{2 M} S_{L T}^{\alpha} \not h, \quad \gamma_{T \mu} T^{\mu \alpha} \not h=\left(\frac{1}{3} S_{L L} \gamma_{T}^{\alpha}+\frac{1}{2} \gamma_{T \mu} S_{T T}^{\mu \alpha}\right) \not h .
$$

As for independent $T^{\mu \nu}$ terms where the index $\alpha$ comes from other vectors, we have two possibilities by noting the transverse components $\epsilon_{T}^{\alpha \nu}\left(\epsilon_{T}^{11}=-\epsilon_{T}^{22}=1\right)$ and $\gamma_{T}^{\alpha}$ as

$$
n_{\mu} T^{\mu \nu} \epsilon_{T \nu}^{\alpha} i \gamma_{5} \not h=\frac{P^{+}}{2 M} \epsilon_{T}^{\alpha \mu} S_{L T \mu} i \gamma_{5} \not h, \quad n_{\mu} T^{\mu \nu} n_{\nu} \gamma_{T}^{\alpha} \not h=\frac{2}{3} S_{L L} \frac{\left(P^{+}\right)^{2}}{M^{2}} \gamma_{T}^{\alpha} \not h .
$$

From these considerations, the correlation function $\Phi_{G}^{\alpha}\left(x_{1}, x_{2}\right)$ of eq. (3.29) is generally expressed by four terms with the tensor polarizations as

$$
\begin{aligned}
\Phi_{G}^{\alpha}\left(x_{1}, x_{2}\right)=\frac{M}{2}[ & i S_{L T}^{\alpha} F_{G, L T}\left(x_{1}, x_{2}\right)-\epsilon_{T}^{\alpha \mu} S_{L T \mu} \gamma_{5} G_{G, L T}\left(x_{1}, x_{2}\right) \\
& \left.+i S_{L L} \gamma^{\alpha} H_{G, L L}^{\perp}\left(x_{1}, x_{2}\right)+i S_{T T}^{\alpha \mu} \gamma_{\mu} H_{G, T T}\left(x_{1}, x_{2}\right)\right]
\end{aligned}
$$

All the terms in the right-hand side of eq. (3.32) satisfy the parity and time-reversal invariances. The functions $F_{G, L T}\left(x_{1}, x_{2}\right), G_{G, L T}\left(x_{1}, x_{2}\right), H_{G, L L}^{\perp}\left(x_{1}, x_{2}\right), H_{G, T T}\left(x_{1}, x_{2}\right)$ are twist-3 multiparton distribution functions. The correlation function $\Phi_{A}^{\alpha}$ is defined by using the gluon field $A^{\alpha}$ instead of the field thensor $G^{+\alpha}$ in eq. (3.29), and it is expressed by real multiparton correlation functions. Therefore, the right-hand side of eq. (3.32) contains the $i$ factor due to the derivative $\partial^{+}\left(\rightarrow i P^{+}\right)$in $G^{+\alpha}$.

We try to find properties of the multiparton distribution functions $F_{G, L T}\left(x_{1}, x_{2}\right)$, $G_{G, L T}\left(x_{1}, x_{2}\right), H_{G, L L}^{\perp}\left(x_{1}, x_{2}\right), H_{G, T T}\left(x_{1}, x_{2}\right)$ under the exchange of variables $x_{1}$ and $x_{2}$ by using the Hermiticity condition. For example, this Hermiticity relation is given for the first term of eq. (3.32) as $-i S_{L T}^{\nu} \not^{\dagger} F_{G, L T}\left(x_{1}, x_{2}\right)^{*}=i S_{L T}^{\nu} \gamma^{0} \not h \gamma^{0} F_{G, L T}\left(x_{2}, x_{1}\right)$. For the real function of $F_{G, L T}\left(x_{1}, x_{2}\right)$, it becomes $F_{G, L T}\left(x_{1}, x_{2}\right)=-F_{G, L T}\left(x_{2}, x_{1}\right)$. In this way, the Hermiticity condition is satisfied if the functions $F_{G, L T}, G_{G, L T}, H_{G, L L}^{\perp}$, and $H_{G, T T}$ are real and they have the properties

$$
\begin{array}{ll}
F_{G, L T}\left(x_{1}, x_{2}\right)=-F_{G, L T}\left(x_{2}, x_{1}\right), & G_{G, L T}\left(x_{1}, x_{2}\right)=G_{G, L T}\left(x_{2}, x_{1}\right), \\
H_{G, L L}^{\perp}\left(x_{1}, x_{2}\right)=H_{G, L L}^{\perp}\left(x_{2}, x_{1}\right), & H_{G, T T}\left(x_{1}, x_{2}\right)=H_{G, T T}\left(x_{2}, x_{1}\right),
\end{array}
$$

under the exchange of the variables $x_{1}$ and $x_{2}$. If the multiparton distribution functions are defined by taking the gluon field $A^{\alpha}$ instead of $G^{+\alpha}$ in eq. (3.29) and the functions 
$\left(F_{A, L T}, G_{A, L T}, H_{A, L L}^{\perp}\right.$, and $\left.H_{A, T T}\right)$ are defined without the $i$ factors, these symmetric properties have opposite signs, namely $F_{A, L T}\left(x_{1}, x_{2}\right)=F_{A, L T}\left(x_{2}, x_{1}\right), G_{A, L T}\left(x_{1}, x_{2}\right)=$ $-G_{A, L T}\left(x_{2}, x_{1}\right), H_{A, L L}^{\perp}\left(x_{1}, x_{2}\right)=-H_{A, L L}^{\perp}\left(x_{2}, x_{1}\right)$, and $H_{A, T T}\left(x_{1}, x_{2}\right)=-H_{A, T T}\left(x_{2}, x_{1}\right)$.

From eqs. (3.29) and (3.32), the functions $F_{G, L T}$ and $G_{G, L T}$ are expressed as the correlation matrix-element forms as

$$
\begin{aligned}
S_{L T}^{\nu} F_{G, L T}\left(x_{1}, x_{2}\right)=-\frac{i}{2 M} g \int \frac{d \xi_{1}^{-}}{2 \pi} \frac{d \xi_{2}^{-}}{2 \pi} e^{i x_{1} P^{+} \xi_{1}^{-}} e^{i\left(x_{2}-x_{1}\right) P^{+} \xi_{2}^{-}} \\
\times\left\langle P, T\left|\bar{\psi}(0) \not h n_{\mu} G^{\mu \nu}\left(\xi_{2}^{-}\right) \psi\left(\xi_{1}^{-}\right)\right| P, T\right\rangle
\end{aligned}
$$

and

$$
\begin{aligned}
S_{L T}^{\nu} G_{G, L T}\left(x_{1}, x_{2}\right)=\frac{i}{2 M} & g \int \frac{d \xi_{1}^{-}}{2 \pi} \frac{d \xi_{2}^{-}}{2 \pi} e^{i x_{1} P^{+} \xi_{1}^{-}} e^{i\left(x_{2}-x_{1}\right) P^{+} \xi_{2}^{-}} \\
& \times\left\langle P, T\left|\bar{\psi}(0) i \gamma_{5} \not n_{\mu} \tilde{G}^{\mu \nu}\left(\xi_{2}^{-}\right) \psi\left(\xi_{1}^{-}\right)\right| P, T\right\rangle .
\end{aligned}
$$

Equations (3.34) and (3.35) were obtained by the traces of $\left(\Phi_{G}^{\alpha}\right)_{i j}\left(x_{1}, x_{2}\right)(\not h)_{j i}$ and $\left(\Phi_{G}^{\alpha}\right)_{i j}\left(x_{1}, x_{2}\right)\left(i \gamma_{5} \not h\right)_{j i}$, respectively. Then, the relations $\epsilon_{T}^{\alpha \mu} S_{L T \mu}=g^{\alpha 1} S_{L T}^{2}-g^{\alpha 2} S_{L T}^{1}$ and $n_{\mu} \tilde{G}^{\beta \gamma} \epsilon_{\beta \gamma}{ }_{\beta \alpha}=g^{\alpha 1} 2 \tilde{G}^{+2}-g^{\alpha 2} 2 \tilde{G}^{+1}$ were used to reach to eq. (3.35). Defining the variable $t$ by $\xi_{2}^{-}=t \xi_{1}^{-}$and calculating derivatives of $F_{G, L T}\left(x_{1}, x_{2}\right)$ and $G_{G, L T}\left(x_{1}, x_{2}\right)$ with respect to $x_{1}$ and $x_{2}$, we obtain the relation with the field tensors $G^{\alpha \mu}$ and $\tilde{G}^{\alpha \mu}$ in eq. (3.28). In this calculation, the principal integral expressed by the sign function

$$
\frac{i}{\pi} \mathscr{P} \int_{-\infty}^{\infty} d \omega \frac{1}{\omega} e^{-i \omega z}=\epsilon(z)=\left\{\begin{array}{ll}
+1 & \text { for } z>0 \\
-1 & \text { for } z<0
\end{array},\right.
$$

is used. The integral region of $x_{2}$ is from -1 to 1 ; however, the integrand with the distribution functions vanish in the region $\left|x_{2}\right| \geq 1$, so that the integral region is extended to the one from $-\infty$ to $\infty$. Here, $\mathscr{P}$ indicates the principal integral. Using eqs. (3.34) and (3.35), we obtain the matrix element of the field tensors in the right-hand side of eq. (3.28) as

$$
\begin{gathered}
\int \frac{d(P \cdot \xi)}{2 \pi} e^{i x_{1} P \cdot \xi}\left\langle P, T\left|g \int_{0}^{1} d t \bar{\psi}(0)\left[i\left(t-\frac{1}{2}\right) G^{\alpha \mu}(t \xi)-\frac{1}{2} \gamma_{5} \tilde{G}^{\alpha \mu}(t \xi)\right] \xi_{\mu} \xi \psi(\xi)\right| P, T\right\rangle_{\xi^{+}=\vec{\xi}_{T}=0} \\
=-2 M S_{L T}^{\nu} \mathscr{P} \int_{-1}^{1} d x_{2} \frac{1}{x_{1}-x_{2}}\left[\frac{\partial}{\partial x_{1}}\left\{F_{G, L T}\left(x_{1}, x_{2}\right)+G_{G, L T}\left(x_{1}, x_{2}\right)\right\}\right. \\
\left.+\frac{\partial}{\partial x_{2}}\left\{F_{G, L T}\left(x_{2}, x_{1}\right)+G_{G, L T}\left(x_{2}, x_{1}\right)\right\}\right] .
\end{gathered}
$$

In this way, it becomes possible to separate the twist-3 effects expressed by the multiparton distribution functions from the twist-2 ones, which is essential in deriving the WW-like relation for the distribution function $f_{L T}$.

The multiparton correction functions $\bar{\Phi}_{G}^{\alpha}$ and $\Phi_{G}^{\alpha, C}$ are defined for antiquarks in the same way with eqs. (3.9), (3.10), and (3.11) by considering the definition for quarks in eq. (3.29). The only extra factor is the gluon tensor $G^{+\alpha}$. Noting the charge-conjugation property for the gluon field $A_{\mu}^{C}=-A_{\mu}$, we find the relation which has the opposite sign of eq. (3.11) as

$$
\Phi_{G}^{\alpha, C}\left(x_{1}, x_{2}, P, T\right)=C\left[\bar{\Phi}_{G}^{\alpha}\left(x_{1}, x_{2}, P, T\right)\right]^{\mathscr{T}} C^{\dagger}
$$


Therefore, the relations between the multiparton correlation functions for antiquarks have different signs from eq. (3.12) as

$$
\Phi_{G}^{\alpha, C[\Gamma]}\left(x_{1}, x_{2}\right)=\left\{\begin{array}{ll}
-\bar{\Phi}_{G}^{\alpha,[\Gamma]}\left(x_{1}, x_{2}\right)=+\Phi_{G}^{\alpha,[\Gamma]}\left(-x_{2},-x_{1}\right) & \text { for } \Gamma=\gamma^{\mu}, \sigma^{\mu \nu}, i \gamma_{5} \sigma^{\mu \nu} \\
+\bar{\Phi}_{G}^{\alpha,[\Gamma]}\left(x_{1}, x_{2}\right)=-\Phi_{G}^{\alpha,[\Gamma]}\left(-x_{2},-x_{1}\right) & \text { for } \Gamma=1, i \gamma_{5}, \gamma_{5} \gamma^{\mu}
\end{array} .\right.
$$

Then, the multiparton distribution functions for antiquark are obtained by noting these correlation-function relations as

$$
\begin{array}{ll}
\bar{F}_{G, L T}\left(x_{1}, x_{2}\right)=F_{G, L T}\left(-x_{2},-x_{1}\right), & \bar{G}_{G, L T}\left(x_{1}, x_{2}\right)=-G_{G, L T}\left(-x_{2},-x_{1}\right), \\
\bar{H}_{G, L L}^{\perp}\left(x_{1}, x_{2}\right)=H_{G, L L}^{\perp}\left(-x_{2},-x_{1}\right), & \bar{H}_{G, T T}\left(x_{1}, x_{2}\right)=H_{G, T T}\left(-x_{2},-x_{1}\right) .
\end{array}
$$

Therefore, the multiparton distribution functions for antiquarks are described by the functions for quarks at negative $x_{1}$ and $x_{2}$; however, they are also expressed by the functions $\bar{F}_{G, L T}, \bar{G}_{G, L T}, \bar{H}_{G, L L}^{\perp}$, and $\bar{H}_{G, T T}$. Similar relations were given in the multiparton distribution functions for the spin-1/2 nucleons in ref. [56].

\subsection{Twist-2 relation and sum rule}

After all these preparations on the tensor-polarized PDFs of spin-1 hadrons, we are now ready to drive a twist-2 relation and a sum rule for the twist-3 distribution function $f_{L T}$. First, the matrix element of the nonlocal operator $\bar{\psi}(0)\left(\partial^{\mu} \gamma^{\alpha}-\partial^{\alpha} \gamma^{\mu}\right) \psi(\xi)$ with the transverse index $\alpha$ was expressed in terms of the tensor-polarized PDFs $f_{1 L L}(x)$ and $f_{L T}(x)$ in eq. (3.17). Second, this nonlocal operator was expressed by the field tensor $G^{\alpha \mu}$ and its dual one $\tilde{G}^{\alpha \mu}$ in eq. (3.28). Third, the matrix element of the field tensors was given by the twist3 multiparton distribution functions $G$ and $F$ in eq. (3.37). Combining eqs. (3.17), (3.28), and (3.37), we obtain

$$
x \frac{d f_{L T}(x)}{d x}=-\frac{3}{2} f_{1 L L}(x)-f_{L T}^{(H T)}(x),
$$

where the twist-3 multiparton-distribution part is defined as

$$
\begin{aligned}
f_{L T}^{(H T)}(x)=-\mathscr{P} \int_{-1}^{1} d y \frac{1}{x-y}[ & \frac{\partial}{\partial x}\left\{F_{G, L T}(x, y)+G_{G, L T}(x, y)\right\} \\
& \left.+\frac{\partial}{\partial y}\left\{F_{G, L T}(y, x)+G_{G, L T}(y, x)\right\}\right] .
\end{aligned}
$$

Here, $H T$ indicates the higher twist. Integrating eq. (3.41) over $x$, we obtain

$$
f_{L T}(x)=\frac{3}{2} \int_{x}^{\epsilon(x)} \frac{d y}{y} f_{1 L L}(y)+\int_{x}^{\epsilon(x)} \frac{d y}{y} f_{L T}^{(H T)}(y),
$$

by using the sign function of eq. (3.36). Namely, the integral is from $x$ to 1 if $x$ is positive for the quark distribution, and it is from $x$ to -1 if $x$ is negative for the antiquark distribution. In eq. (3.43), we obtained the full decomposition $f_{L T}$ into the twist-2 term and the twist-3 multiparton distribution functions, as investigated for $g_{2}$ and $h_{L}$ in refs. [32, 42-45]. 
In section 2, the structure function $g_{1}$ is given by the function $g_{1 L}$ as $g_{1}(x)=\left[g_{1 L}(x)+\right.$ $\left.g_{1 L}(-x)\right] / 2$ for describing both the quark and antiquark distributions in the $x$ range of $0 \leq x \leq 1$. In the same way, we define the distribution functions $f_{1 L L}^{+}, f_{L T}^{+}$, and $f_{L T}^{(H T)+}$ as

$$
f^{+}(x) \equiv f(x)+\bar{f}(x)=f(x)-f(-x), \quad f=f_{1 L L}, f_{L T}, f_{L T}^{(H T)}, \quad x>0 .
$$

Here, these functions are given for a single flavor. From eqs. (3.43) and (3.44), we obtain a relation for $f_{L T}^{+}(x)$ and $f_{1 L L}^{+}(x)$ as

$$
f_{L T}^{+}(x)=\frac{3}{2} \int_{x}^{1} \frac{d y}{y} f_{1 L L}^{+}(y)+\int_{x}^{1} \frac{d y}{y} f_{L T}^{(H T)+}(y) .
$$

Since the function $f_{L T}^{(H T)+}(y)$ indicates the twist-3 effects as given by the multiparton correlation functions, this equation indicates that the twist- 2 part of the function $f_{L T}^{+}(x)$ is expressed by the integral of $f_{1 L L}^{+}(x)$. In this way, the twist-3 function $f_{L T}^{+}(x)$ is expressed by the twist-2 function and the remaining twist-3 one. If the twist-3 part is neglected, the relation becomes

$$
f_{L T}^{+}(x)=\frac{3}{2} \int_{x}^{1} \frac{d y}{y} f_{1 L L}^{+}(y) .
$$

This equation corresponds to the WW relation in eq. (2.12). It should be noted that the structure function $b_{1}$ or the tensor-polarized PDF $\delta_{T} q$ is given by the $f_{1 L L}$ as $-(3 / 2) f_{1 L L}^{+}=$ $b_{1}^{q}+b_{1}^{\bar{q}}=\delta_{T} q+\delta_{T} \bar{q}[25]$. This new relation suggests that the tensor-polarized distribution function $f_{L T}(x)$ is expressed by the integral of $f_{1 L L}(x)$ or $b_{1}(x)$ if higher-twist effects are ignored. If the function $f_{2 L T}(x)$ is defined by

$$
f_{2 L T}(x) \equiv \frac{2}{3} f_{L T}(x)-f_{1 L L}(x),
$$

eq. (3.45) becomes

$$
f_{2 L T}^{+}(x)=-f_{1 L L}^{+}(x)+\int_{x}^{1} \frac{d y}{y} f_{1 L L}^{+}(y)+\frac{2}{3} \int_{x}^{1} \frac{d y}{y} f_{L T}^{(H T)}(y) .
$$

The distribution function $f_{2 L L}^{+}(x)$ is expressed by the twist-2 and twist- 3 terms. If the twist-3 term is neglected, we obtain a relation

$$
f_{2 L T}^{+}(x)=-f_{1 L L}^{+}(x)+\int_{x}^{1} \frac{d y}{y} f_{1 L L}^{+}(y),
$$

which is analogous to the WW relation for $g_{1}$ and $g_{2}$ in eq. (2.12). Furthermore, integrating this equation, we obtain

$$
\int_{0}^{1} d x f_{2 L T}^{+}(x)=0
$$

which is analogous to the BC sum rule. These relation and sum rule are useful in studying the tensor-polarized distribution function $f_{2 L T}^{+}(x)$ (or original $f_{L T}(x)$ ) as the WW relation and the $\mathrm{BC}$ sum rule provide strong constraints on determining the structure function 
$g_{2}(x)$ for the nucleons. Furthermore, considering the sum rule based on the parton model $\int d x b_{1}(x)=0$ (or $\int d x f_{1 L L}^{+}(x)=0$ by the notation in this paper) [3-5], which is valid if tensor-polarized antiquark distributions vanish, we have the sum rule for the twist-3 function $f_{L T}$ itself as

$$
\int_{0}^{1} d x f_{L T}^{+}(x)=0
$$

Equations (3.50) and (3.51) could be affected by the small- $x$ behavior of the distribution functions in the same way with the BC sum rule for $g_{2}$. We proved these twist-2 relations in the tree level and have not discussed perturbative QCD corrections. At this stage, it is not obvious whether these relations are satisfied in the structure-function level by including coefficient functions as investigated in $g_{2}$ [35]. We leave this issue for a future project.

The tensor-polarized structure functions of spin-1 hadrons and nuclei have been investigated since the end of 1980's. Due to lack of experimental measurements except for the HERMES experiment for $b_{1}$, theoretical developments of this field were rather slow in comparison with the spin physics of spin- $1 / 2$ nucleons. However, we believe that bright future is ahead of us in the sense that the tensor-polarized structure function $b_{1}$ and the gluon transversity, which are specific observables in the spin-1 hadrons, will be measured at JLab in the middle of 2020's [7, 9, 10] and such experiments will be proposed at Fermilab [19] as the proton-deuteron Drell-Yan process. The NICA facility will have the polarized-deuteron beam in the near future [21, 22], so that they could focus their studies on the structure functions of the spin-1 deuteron. In addition, there are EIC projects in US and China [12, 13] to investigate the structure functions of the spin-1 hadrons and nuclei in 2030's. In the JLab measurements, the scale $Q^{2}$ is not large in general, which enables to probe the twist-3 structure functions such as $g_{2}$. In the same way, higher-twist tensor-polarized structure functions could become accessible at JLab or future high-intensity accelerator facilities. In this sense, our previous studies on general twist-3 and twist-4 distribution functions [25] as well as this work should become useful in future. In particular, the new twist-2 relation of eq. (3.49) [or (3.46)] and the sum rule of eq. (3.50) [also eq. (3.51) in addition] could become important for constraining the twist-3 function $f_{2 L T}(x)$ or $f_{L T}(x)$, although the integral relations are always difficult to be tested due to the experimental inaccessibility at small $x$. The tensor-polarized PDFs and structure functions will be measured at JLab, Fermilab, and NICA at relatively large $x$, and the small- $x$ part will be investigated at EIC and other high-energy lepton facilities, for example, by a possible fixed target project of a linear or circular lepton collider. According to the theoretical estimate on higher-twist tensor-polarized structure functions in a few- $\mathrm{GeV} Q^{2}$ region [8], which is the typical kinematical region of JLab, they are not be much smaller than the leading-twist ones, in the similar way to the $15-40 \%$ breaking of the WW relation for $g_{2}[47,48]$. Therefore, the understanding of the higher-twist structure functions are valuable also in determining the leading-twist functions from actual measurements in future. At this stage, the available information is very limited for the tensor-polarized PDFs and structure functions. However, we hope to make progress on numerical studies of them by considering the sum rules derived in this work. 


\section{Summary}

There are tensor-polarized PDFs and structure functions for spin-1 hadrons. In this work, we derived a new useful twist-2 relation

$$
f_{L T}(x)=\frac{3}{2} \int_{x}^{\epsilon(x)} d y \frac{f_{1 L L}(y)}{y}+\int_{x}^{\epsilon(x)} d y \frac{f_{L T}^{(H T)}(y)}{y},
$$

where $\epsilon(x)=1(-1)$ at $x>0(x<0)$, for the twist-3 distribution function $f_{L T}(x)$ and twist-2 one $f_{1 L L}(x)$. This equation indicates the quark and antiquark distributions at $x>0$ and $x<0$, respectively. Defining the plus function by the quark and antiquark distributions as $f^{+}(x)=f(x)+\bar{f}(x)$ and neglecting the higher-twist term, this equation is written as

$$
f_{L T}^{+}(x)=\frac{3}{2} \int_{x}^{1} \frac{d y}{y} f_{1 L L}^{+}(y) .
$$

Namely, the twist-2 part of $f_{L T}(x)$ is expressed by the integral of $f_{1 L L}(x)$. Since the integrand is given by the structure function $b_{1}^{q+\bar{q}}=-(3 / 2) f_{1 L L}^{+}$, the twist- 2 of $f_{L T}(x)$ is expressed by the function $b_{1}$. Using the function $f_{2 L L}$ defined by $f_{2 L L}=\frac{2}{3} f_{L T}-f_{1 L L}$, we obtained

$$
f_{2 L T}^{+}(x)=-f_{1 L L}^{+}(x)+\int_{x}^{1} \frac{d y}{y} f_{1 L L}^{+}(y) .
$$

This relation is similar to the Wandzura-Wilczek relation for the polarized structure functions $g_{1}$ and $g_{2}$ for the spin- $1 / 2$ nucleons. It is useful in the sense that the twist- 2 part is constrained and the separation of higher-twist effects become clear. In addition, we showed that the sum rule

$$
\int_{0}^{1} d x f_{2 L T}^{+}(x)=0
$$

exists for $f_{2 L L}$, and it constrains the overall $x$-dependent functional form of $f_{2 L L}$. It is similar to the Burkhardt-Cottingham sum rule for $g_{2}$. Furthermore, if the parton-model sum rule $\int d x f_{1 L L}^{+}(x)=0\left(\int d x b_{1}^{q+\bar{q}}(x)=0\right)$ is applied in the case where the tensorpolarized antiquark distributions vanish, it led to another sum rule

$$
\int_{0}^{1} d x f_{L T}^{+}(x)=0
$$

All these relations are valuable in investigating the tensor-polarized PDFs and structure functions in future. For specifying twist-3 terms in deriving these relations, we explained that four twist-3 multiparton distribution functions

$$
F_{L T}\left(x_{1}, x_{2}\right), \quad G_{L T}\left(x_{1}, x_{2}\right), \quad H_{L L}^{\perp}\left(x_{1}, x_{2}\right), \quad H_{T T}\left(x_{1}, x_{2}\right),
$$

exist for tensor-polarized spin-1 hadrons. These multiparton distribution functions are also interesting for probing multiparton correlations in spin-1 hadrons. 


\section{Acknowledgments}

The authors thank N. Sato for suggestions. S. Kumano was partially supported by Japan Society for the Promotion of Science (JSPS) Grants-in-Aid for Scientific Research (KAKENHI) Grant Number 19K03830. Qin-Tao Song was supported by the National Natural Science Foundation of China under Grant Number 12005191 and the Academic Improvement Project of Zhengzhou University.

Open Access. This article is distributed under the terms of the Creative Commons Attribution License (CC-BY 4.0), which permits any use, distribution and reproduction in any medium, provided the original author(s) and source are credited.

\section{References}

[1] L.L. Frankfurt and M.I. Strikman, High-momentum-transfer processes with polarized deuterons, Nucl. Phys. A 405 (1983) 557 [inSPIRE].

[2] P. Hoodbhoy, R.L. Jaffe and A. Manohar, Novel Effects in Deep Inelastic Scattering from Spin 1 Hadrons, Nucl. Phys. B 312 (1989) 571 [InSPIRE].

[3] F.E. Close and S. Kumano, Sum rule for the spin-dependent structure function $b_{1}(x)$ for spin-one hadrons, Phys. Rev. D 42 (1990) 2377 [INSPIRE].

[4] S. Kumano, Tensor-polarized structure functions: Tensor structure of deuteron in 2020's, J. Phys. Conf. Ser. 543 (2014) 012001 [arXiv: 1407.3852] [INSPIRE].

[5] HERMES collaboration, Measurement of the Tensor Structure Function $b_{1}$ of the Deuteron, Phys. Rev. Lett. 95 (2005) 242001 [hep-ex/0506018] [InSPIRE].

[6] S. Kumano, Tensor-polarized quark and antiquark distribution functions in a spin-one hadron, Phys. Rev. D 82 (2010) 017501 [arXiv: 1005.4524] [INSPIRE].

[7] J.-P. Chen et al., The Deuteron Tensor Structure Function b $b_{1}$, Proposal to Jefferson Lab PAC-38, PR12-11-110 (2011) https://www.jlab.org/exp_prog/proposals/11/PR12-11-110.pdf.

[8] W. Cosyn, Y.-B. Dong, S. Kumano and M. Sargsian, Tensor-polarized structure function $b_{1}$ in standard convolution description of deuteron, Phys. Rev. D 95 (2017) 074036 [arXiv: 1702.05337] [INSPIRE].

[9] R.L. Jaffe and A. Manohar, Nuclear gluonometry, Phys. Lett. B 223 (1989) 218 [InSPIRE].

[10] M. Jones et al., Search for Exotic Gluonic States in the Nucleus, A Letter of Intent to Jefferson Lab PAC 44, LOI12-16-006 (2016)

https://www.jlab.org/exp_prog/proposals/16/LOI12-16-006.pdf.

[11] J.P. Ma, C. Wang and G.P. Zhang, Azimuthal Dependence of DIS with Spin-1 Target,

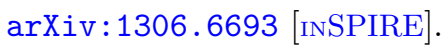

[12] R. Abdul Khalek et al., Science Requirements and Detector Concepts for the Electron-Ion Collider: EIC Yellow Report, arXiv:2103.05419 [INSPIRE].

[13] D.P. Anderle et al., Electron-ion collider in China, Front. Phys. (Beijing) 16 (2021) 64701 [arXiv: 2102.09222] [INSPIRE]. 
[14] S. Hino and S. Kumano, Structure functions in the polarized Drell-Yan processes with spin-1/2 and spin-1 hadrons. 1. General formalism, Phys. Rev. D 59 (1999) 094026 [hep-ph/9810425] [INSPIRE].

[15] S. Hino and S. Kumano, Structure functions in the polarized Drell-Yan processes with spin-1/2 and spin-1 hadrons. 2. Parton model, Phys. Rev. D 60 (1999) 054018 [hep-ph/9902258] [INSPIRE].

[16] S. Kumano and Q.-T. Song, Theoretical estimate on tensor-polarization asymmetry in proton-deuteron Drell-Yan process, Phys. Rev. D 94 (2016) 054022 [arXiv:1606.03149] [INSPIRE].

[17] S. Kumano and Q.-T. Song, Gluon transversity in polarized proton-deuteron Drell-Yan process, Phys. Rev. D 101 (2020) 054011 [arXiv:1910.12523] [INSPIRE].

[18] S. Kumano and Q.-T. Song, Deuteron polarizations in the proton-deuteron Drell-Yan process for finding the gluon transversity, Phys. Rev. D 101 (2020) 094013 [arXiv:2003.06623] [INSPIRE].

[19] D. Geesaman et al., Letter of Intent for a Drell-Yan experiment with a polarized proton target, Proposal to Fermilab PAC, P-1039 (2013)

https://www.fnal.gov/directorate/program_planning/June2013PACPublic/P_ 1039_LOI_polarized_DY.pdf.

[20] D. Keller, D. Crabb and D. Day, Enhanced tensor polarization in solid-state targets, Nucl. Instrum. Meth. A 981 (2020) 164504 [arXiv:2008.09515] [INSPIRE].

[21] NICA, For the Spin Physics Detector (SPD) project at NICA, http://spd.jinr.ru/.

[22] A. Arbuzov et al., On the physics potential to study the gluon content of proton and deuteron at NICA SPD, Prog. Nucl. Part. Phys. 119 (2021) 103858 [arXiv:2011.15005] [INSPIRE].

[23] G.A. Miller, Pionic and Hidden-Color, Six-Quark Contributions to the Deuteron b1 Structure Function, Phys. Rev. C 89 (2014) 045203 [arXiv:1311.4561] [inSPIRE].

[24] M. Nzar and P. Hoodbhoy, Estimation of the double helicity flip deuteron structure function, Phys. Rev. D 45 (1992) 2264 [inSPIRE].

[25] S. Kumano and Q.-T. Song, Transverse-momentum-dependent parton distribution functions up to twist 4 for spin-1 hadrons, Phys. Rev. D 103 (2021) 014025 [arXiv:2011.08583] [INSPIRE].

[26] A. Bacchetta and P.J. Mulders, Deep inelastic leptoproduction of spin-one hadrons, Phys. Rev. D 62 (2000) 114004 [hep-ph/0007120] [INSPIRE].

[27] A. Bacchetta, Probing the Transverse Spin of Quarks in Deep Inelastic Scattering, Ph.D. thesis, Vrije University, Amsterdam (2002) hep-ph/0212025 [INSPIRE].

[28] T.A.A. van Daal, Mapping the internal structure of hadrons through color and spin effects, Ph.D. thesis, Groningen Univesity (2018) arXiv:1812.07336 [INSPIRE].

[29] S. Cotogno, Polarized partons in hadrons at high energy, Ph.D. thesis, Free University, Amsterdam (2018) [INSPIRE].

[30] S. Wandzura and F. Wilczek, Sum Rules for Spin Dependent Electroproduction: Test of Relativistic Constituent Quarks, Phys. Lett. B 72 (1977) 195 [INSPIRE].

[31] H. Burkhardt and W.N. Cottingham, Sum rules for forward virtual Compton scattering, Annals Phys. 56 (1970) 453 [INSPIRE]. 
[32] J. Kodaira and K. Tanaka, Polarized structure functions in QCD, Prog. Theor. Phys. 101 (1999) 191 [hep-ph/9812449] [INSPIRE].

[33] J. Blümlein and N. Kochelev, On the twist -2 and twist - three contributions to the spin dependent electroweak structure functions, Nucl. Phys. B 498 (1997) 285 [hep-ph/9612318] [INSPIRE].

[34] J. Blümlein and A. Tkabladze, Target mass corrections for polarized structure functions and new sum rules, Nucl. Phys. B 553 (1999) 427 [hep-ph/9812478] [INSPIRE].

[35] V.M. Braun, G.P. Korchemsky and A.N. Manashov, Evolution equation for the structure function $g_{2}\left(x, Q^{2}\right)$, Nucl. Phys. B 603 (2001) 69 [hep-ph/0102313] [INSPIRE].

[36] M. Anselmino, A. Efremov and E. Leader, The Theory and phenomenology of polarized deep inelastic scattering, Phys. Rept. 261 (1995) 1 [Erratum ibid. 281 (1997) 399] [hep-ph/9501369] [INSPIRE].

[37] B. Lampe and E. Reya, Spin physics and polarized structure functions, Phys. Rept. 332 (2000) 1 [hep-ph/9810270] [INSPIRE].

[38] E. Leader, Spin in Particle Physics, Cambridge University Press (2001) [DOI].

[39] R.L. Jaffe and X.-D. Ji, Studies of the transverse spin-dependent structure function $g_{2}\left(x, Q^{2}\right)$, Phys. Rev. D 43 (1991) 724 [inSPIRE].

[40] R.L. Jaffe, Spin, twist and hadron structure in deep inelastic processes, in Ettore Majorana International School of Nucleon Structure: 1st Course: The Spin Structure of the Nucleon, (1996), pp. 42-129 [hep-ph/9602236] [INSPIRE].

[41] C. Itzkyson and J.-B. Zuber, Quantum Field Theory McGraw-Hill Inc. (1980).

[42] R.L. Jaffe and X.-D. Ji, Chiral-odd parton distributions and polarized Drell-Yan process, Phys. Rev. Lett. 67 (1991) 552 [InSPIRE].

[43] R.L. Jaffe and X.-D. Ji, Chiral odd parton distributions and Drell-Yan processes, Nucl. Phys. B 375 (1992) 527 [INSPIRE].

[44] A.V. Belitsky and D. Mueller, Scale dependence of the chiral-odd twist-3 distributions $h_{L}(x)$ and e(x), Nucl. Phys. B 503 (1997) 279 [hep-ph/9702354] [INSPIRE].

[45] A.V. Belitsky, Leading order analysis of twist-3 space- and time-like cut vertices in QCD, Int. J. Mod. Phys. A 32 (2017) 1730018 [hep-ph/9703432] [InSPIRE].

[46] V. Barone and R.G. Ratcliffe, Transverse Spin Physics, World Scientific, Singapore (2003) [DOI].

[47] A. Accardi, A. Bacchetta, W. Melnitchouk and M. Schlegel, What can break the Wandzura-Wilczek relation?, JHEP 11 (2009) 093 [arXiv:0907.2942] [INSPIRE].

[48] Jefferson Lab Angular Momentum collaboration, Iterative Monte Carlo analysis of spin-dependent parton distributions, Phys. Rev. D 93 (2016) 074005 [arXiv:1601.07782] [INSPIRE].

[49] A. Deur, S.J. Brodsky and G. F de Teramond, The spin structure of the nucleon, Rept. Prog. Phys. 82 (2019) 076201 [arXiv: 1801.09154] [INSPIRE].

[50] I.I. Balitsky and V.M. Braun, Evolution Equations for QCD String Operators, Nucl. Phys. B 311 (1989) 541 [INSPIRE]. 
[51] T. Rogers, Transverse moments of TMD parton densities and ultraviolet divergences, Mod. Phys. Lett. A 35 (2020) 37. [arXiv:2008.05351] [InSPIRE]

[52] P.J. Mulders, Lectures at the 17th Taiwan Nuclear Physics School, Institute of Physics, Academia Sinica, Taipei, August 25-28, 2014, http://www.nat.vu.nl/ mulders/.

[53] R.D. Tangerman, Higher-twist correlations in polarized hadrons, Ph.D. thesis, Free University Amsterdam (1996),

https://inis.iaea.org/collection/NCLCollectionStore/_Public/28/015/28015381.pdf.

[54] P. Ball and V.M. Braun, Higher twist distribution amplitudes of vector mesons in QCD: twist-4 distributions and meson mass corrections, Nucl. Phys. B 543 (1999) 201 [hep-ph/9810475] [INSPIRE].

[55] S.J. Brodsky, H.-C. Pauli and S.S. Pinsky, Quantum chromodynamics and other field theories on the light cone, Phys. Rept. 301 (1998) 299 [hep-ph/9705477] [INSPIRE].

[56] K. Kanazawa, Y. Koike, A. Metz, D. Pitonyak and M. Schlegel, Operator constraints for twist-3 functions and Lorentz invariance properties of twist-3 observables, Phys. Rev. D 93 (2016) 054024 [arXiv: 1512.07233] [INSPIRE]. 